\title{
Comparação de métodos para avaliação de beta-hidroxibutirato em ovelhas
}

Luiza Rodegheri Jacondino ${ }^{[a]}$, Andressa Silveira Gonçalves ${ }^{[a]}$, Priscila Teixeira Ferreirad ${ }^{[a]}$, Beatriz Riet Correa Rivero ${ }^{[a]}$, Eneder Rosana Oberst ${ }^{[a]}$, Marcos Kipper Silva ${ }^{[b]}$, Ines Andretta ${ }^{[b]}$, Daniela Becker Birge ${ }^{[c]}$, Eduardo Harry Birgel Junior ${ }^{[c]}$, Raquel Fraga e Silva Raimondo $0^{[]^{0}}$

\footnotetext{
[a] Núcleo RuminAção-Ensino, Pesquisa e Extensão em Ruminantes, Faculdade de Veterinária, Universidade Federal do Rio Grande do Sul (UFRGS), Porto Alegre, RS, Brasil

[b] Departamento de Zootecnia, Faculdade de Agronomia, Universidade Federal do Rio Grande do Sul (UFRGS), Porto Alegre, RS, Brasil

[c] Departamento de Medicina Veterinária, Faculdade de Zootecnia e Engenharia de Alimentos, Universidade de São Paulo (USP), São Paulo, SP, Brasil
}

*Autor correspondente

e-mail: rfraimondo@gmail.com

\section{Resumo}

O final da gestação e o início da lactação correspondem ao período de maior exigência metabólica e, consequentemente, aumento do risco de toxemia da prenhez e cetose em ovelhas. Nessa fase, ocorre grande variação dos metabólitos sanguíneos, entre eles beta-hidroxibutirato ( $\beta$-HBO). Fêmeas consideradas com risco eminente de desenvolverem a doença possuem valores de $\beta$-HBO sanguíneo entre 0,60 e 0,80 mmol/L. Considera-se o valor de 0,8 mmol/L e 1,6 mmol/L como indicativos de cetose inaparente e cetose clínica em ovinos, respectivamente. As técnicas mais precisas de mensuração de corpos cetônicos exigem estrutura laboratorial apropriada, aumentando os custos e impossibilitando a execução a campo. Atualmente, o uso de sensores portáteis de uso humano e uso veterinário específico para mensuração da cetose em vacas está padronizado e difundido na rotina clínica, contudo, estudos em ovinos são escassos. Sendo assim, a presente pesquisa objetivou avaliar a acurácia e precisão do sensor portátil de uso humano Optium Xceed ${ }^{\circledR}$ (Abbott Diabetes CareLtd., Witney, UK) e do sensor protátil KetoVet ${ }^{\circledR}$ (KetoVet Brasil, TaiDoc technology, Taiwan, China) de uso veterinário específico para bovinos para a determinação de $\beta$-HBO em ovelhas no final da gestação e pós-parto recente. Foram utilizadas 37 amostras provenientes de nove ovelhas mestiças Corriedale. As amostras de sangue foram obtidas por punção da veia jugular externa, utilizando-se o sistema Vacutainer ${ }^{\circledR}$ com tubos sem anticoagulante, colhidas semanalmente a partir de 52 dias pré-parto, no dia do parto, 3, 7 e 10 dias após o parto. Imediatamente após a colheita, foi realizada diretamente no tubo de 
coleta a mensuração de $\beta$-HBO no sensor portátil de uso humano e no sensor portátil veterinário. A seguir, as amostras foram centrifugadas a 1000g por 15 minutos, sendo o soro separado e conservado em freezer a menos $20^{\circ} \mathrm{C}$ até a análise. A determinação bioquímica de $\beta$-HBO no soro foi realizada utilizando metodologia enzimática colorimétrica, considerada padrão ouro, com kit comercial (Ranbut D-3-Hydroxibutyrate, Randox Laboratories Ltd, Reino Unido) em analisador bioquímico automático (Labtest modelo Labmax 240, Japão - Tokyo Boeki Medical System Ltda). A média obtida na bioquímica sérica, considerada padrão, foi de 0,497 $\mathrm{mmol} /( \pm 0,214)$; no sensor de uso humano a média foi igual a $0,537 \mathrm{mmol} / \mathrm{L}( \pm 0,332)$, enquanto que no sensor de uso veterinário foi de $0,751 \mathrm{mmol} / \mathrm{L}( \pm 0,367)$. Foi verificada alta correlação entre o dosímetro de uso humano e o padrão ouro $(\mathrm{r}=0,93, \mathrm{P}<0,001)$. A média do aparelho de uso veterinário diferiu das demais, sendo $51 \%$ maior que o padrão, ou seja, foi menos preciso e teve menor veracidade, superestimando os resultados em ovelhas. Concluiu-se que o sensor portátil de uso humano é mais acurado e mais preciso no diagnóstico precoce de toxemia da gestação em ovelhas. 